\title{
Ear Differences and Implied Cerebral Lateralization on Some Intellective Auditory Factors
}

\author{
Lazar Stankov \\ University of Sydney
}

\begin{abstract}
A battery of auditory tests was given under the conditions of monaural and binaural presentation. The results indicated that both primary and second-order factors were similar to those found earlier with the same tests. The hierarchical solution also indicated that most of the differences between the conditions of presentation occurred at the lowest order of factoring. Differences between the means showed the same trends as those reported in the literature on hemispheric specialization. $\mathrm{Ob}$ tained first-order factors were interpreted as Tonal Memory, Speech Perception Under Distraction/Distortion, and Maintaining and Judging Rhythm, all representing a measure of General Auditory Function. In addition, a broad first-order factor of Fluid Intelligence was identified along with Temporal Tracking, representing an interesting new component. Although General Auditory Function is a broad perceptual factor akin to General Visualization, it differs from the latter in an important way. It is suggested that competition between the auditory messages may be typical of General Auditory Function but that the hemispheric localization is not.
\end{abstract}

The theory of Fluid (Gf) and Crystallized (Gc) Intelligence (Cattell, 1971; Horn, 1968) postulates that, in addition to these two, there exist several other broad abilities. One of these, General Visualization (Gv), has been studied extensively in the past. The other, General Auditory

APPLIED PSYCHOLOGICAL MEASUREMENT Vol. 4, No. 1 Winter 1980 pp. 21-38

(C) Copyright 1980 West Publishing Co.
Function (Ga), has been relatively less explored (Stankov, 1971, 1978; Stankov \& Horn, in press).

Both $\mathrm{Gv}$ and $\mathrm{Ga}$ are considered to represent perceptual abilities, as distinct from intellectual abilities of the Gf-Gc variety. Bock (1973) has implied that one of their characteristics is the right-hemisphere localization; and at present, there could be little doubt that $\mathrm{Gv}$ is indeed localized in this way. The aim of this study was to determine whether the same holds for $\mathrm{Ga}$ as well.

The question of hemispheric lateralization has been a subject of considerable interest during the past decade. The following sections will (1) briefly review some of the findings related to the processing of the auditory stimuli; (2) describe primary and second-order auditory factors; and (3) derive hypotheses regarding their lateralization. In the second part of the paper some relevant data will be considered.

\section{Some Findings Regarding Hemispheric Specialization}

Because of the symmetric projections of each ear, it was necessary to adopt special experimental techniques for the purpose of detecting hemispheric differences. For monaural stimulation, reaction time has been used as a dependent 
measure and ear asymmetry has been demonstrated (Haydon \& Spellacy, 1973). "Number correct" can also be used as a dependent measure of lateralization, provided that either delayed feedback (Roberts \& Gregory, 1973) or the dichotic listening paradigm (Kimura, 1967) is employed. Usually, in order to explain this asymmetry, it is assumed (1) that the contralateral auditory pathways have perceptual preference over the ipsilateral and (2) that the left hemisphere is specialized for language or speech and the right hemisphere for certain nonlanguage stimuli (Milner, 1974). According to Kimura's (1967) "structural model," perceptual preference for contralateral pathways implies occlusion of information arriving ipsilaterally. Darwin (1974) elaborated in some detail on Kimura's idea and called it "functional decussation." He also implied that it would be possible to detect ear differences even without resorting to dichotic listening techniques, provided that a semantically loaded task (e.g., Verbal Comprehension) is used.

A general statement that the left hemisphere is responsible for processing speech and that the right hemisphere deals with nonspeech sounds would probably be considered an oversimplification. First, the left hemisphere is also responsible for processing nonspeech rhythmic auditory stimuli (Robinson \& Solomon, 1973), although delayed auditory feedback seems to provide a conflicting result (Roberts \& Gregory, 1973). The right hemisphere is responsible for perception of melodies (Kimura, 1967), chords (Gordon, 1970), performance on Seashore's tests of Timbre and Tonal Memory (Milner, 1962), and so forth. However, Bever and Chiarello (1974) and Gordon (1974) have observed that people, usually with musical training, can be found who demonstrate a right-ear superiority on the melodies test.

Secondly, not all aspects of speech are processed by the left hemisphere. Under many conditions, consonants show a definite right ear, left-hemisphere superiority (Darwin, 1971; Shankweiler \& Studdert-Kennedy, 1967). Vow- els, on the other hand, do not show any ear differences unless the subjects' expectation has been manipulated or the duration of the vowel stimulus has been shortened (Blumstein \& Cooper, 1974). The latter investigators also found that intonation contours of speech are processed by the right hemisphere. ${ }^{1}$

The above-mentioned studies involving auditory stimuli used a variety of tests which would be expected to correlate among themselves and to form a smaller number of auditory factors. The task of determining these factors has already been undertaken and some of the results will be considered in the next section.

\section{Some Primary Auditory Factors}

Stankov (1971), Stankov and Spilsbury (1978), and Stankov and Horn (in press) showed that there are four primaries within the auditory domain that have not been incorporated within the theories of abilities. These primaries are at least as important in real life and as invariant and stable across cultures as some of the visual primaries listed by French, Ekstrom, and Price (1963).

It is convenient to talk about the four auditory primaries in terms of the second-order factors that they define. One of the four loads on $\mathbf{G f}$ and in some ways is probably more important than the others: It has been labeled Temporal Tracking.

When Horn (e.g., 1972) discussed anlage functions representing the basis of Gf, he usually cited two examples. One of these is "span of awareness" and the other is Hearnshaw's (1956) notion of "temporal integration." A measure of

\footnotetext{
'Most of the studies reviewed here and the new data to be presented in this paper are based upon the investigation of an intact brain. A somewhat different tradition in investigating lateralization derives from the studies involving commissurotomized or brain-damaged (aphasic) subjects. Although there is no pronounced discrepancy between the two traditions in terms of basic findings, this paper refers to the latter kind of studies only, in order to derive hypotheses when evidence from the intact-brain studies is lacking.
} 
the first is, of course, a memory span test; but Horn has never properly tested the second notion. Arnold (1964) gave two of Hearnshaw's tests of temporal integration, together with markers for memory and reasoning, to a large sample of high school students. She found that a temporal integration factor could not be identified and was led to conclude that temporal integration tests do not measure any novel factor but show loadings simila: to those of reasoning tests.

That alleged tests of temporal integration apparently do not measure anything new largely undermines the validity of the notion, in spite of its possible intuitive appeal. However, several tests of temporal reordering defined a single factor in all of Stankov's studies. These tests involve listening to three auditory stimuli (tones varying in pitch or loudness, nonsense syllables, letters, different voices saying the same word); remembering the order in which stimuli occur; and when they are given again, writing down the numbers corresponding to their first presentation. For example,

First Presentation: B A C attached order 123

Second Presentation: A B C

Answer: 213.

It is Temporal Tracking (Tc), a Gf primary that differs from Hearnshaw's temporal integration, that is the factor mainly defined by these tests. It seems to correspond to what some contemporary cognitive psychologists (e.g., Hunt, Lunneborg, \& Lewis, 1975) have emphasized as an important part of intelligence, i.e., superior ability at retaining, in short-term memory, information about the order of stimulus presentation, perhaps by means of an internally assigned time tag.

The second primary factor (Discrimination Among Sound Patterns; DASP) had loadings on all three second-order factors (Gf, Gc, Ga) of Stankov's (1971) study and on Gf only in his 1978 study. This factor involves memory for sounds, whether they are musical or linguistic in nature. Several speech tests which loaded on DASP were not included in the Stankov and Spilsbury (1978) study, and only musical memory tests were retained, forming a factor called Tonal Memory (Tm)-a rather common factor, which had been found by Karlin (1941), among others. This time, however, it behaved as a Ga primary. It can be said, then, that inclusion or exclusion of certain speech tests determines whether DASP or the narrower Tm occurs and consequently whether the factor will load on Gc, $\mathrm{Gf}$, or $\mathrm{Ga}$, or on all three.

The third and fourth primary factors are reliable measures of Ga. Maintaining and Judging Rhythm (MaJR) is measured by several tests of rhythm and tempo, which, in one study, showed some involvement of a test that was based on sentences. This is consistent with the stress by cognitive psychologists on rhythm as an important factor in speech perception. Speech Perception Under Distraction/Distortion (SPUD) is defined by tests in which an auditory message has been mutilated (e.g., tests of high and low pass filter, intellective and noise masking, compressed speech, understanding of accents and stutterers). It can be claimed that a person taking all these tests must resist distraction and, sometimes, must persist in working through an unpleasant experience.

In recent literature on cerebral lateralization, there are numerous studies dealing with often minute and certainly rather specific aspects of information processing. Research on individual differences that is based on multivariate methodology provides one way to organize the study of the domain in a rational fashion.

\section{Hypothesis Regarding Lateralization}

On the basis of the evidence presented earlier in this paper, it seems that primaries representing Gc are "localized" in the left hemisphere (see Darwin, 1974). If a particular primary measures Gf, lateralization should not be expected unless the visualization and/or verbal 
component are pronounced in the test material used. This statement is based upon the result of Zaidel and Sperry (1973), who investigated hemispheric lateralization of the Raven's Coloured Progressive Matrices Test within a sample of commisurotomized patients.

It has already been suggested that one of the two broad perceptual factors, $\mathrm{Gv}$, is determined by the functioning of the right cerebral hemisphere. $\mathrm{Ga}$, on the other hand, probably cannot be located in such a simple way; the particular primaries that define it should be examined. With two of the primaries-MaJR and Tm-the task is relatively easy, since previously reviewed studies strongly indicate that $\mathrm{Tm}$ is located on the right and rhythm seems to be localized in the left. However, with SPUD, a simple placement is not possible.

Lateralization here simply means that one ear, and consequently the opposite hemisphere, is superior in performing a particular task. The aim of this paper was to test the above hypotheses. Prior to that, however, an aspect of lateralization that has provoked virtually no attention up to now was examined. This aspect is related to the question of whether factor structure is dependent upon mode of presentation of auditory stimuli, i.e., whether there would be any differences in factor structure if auditory tests were presented through either left, right, or both ears. Without such information, it is wrong to conclude that if there is an identical difference between the hemispheres with regard to two tasks (say, left hemisphere is superior when performing on vocabulary and spelling tests), the left hemisphere is specialized for analytic or parallel processing. Such an assumption derives from comparisons between the means only; and it has yet to be shown that it is not the case that, for example, vocabulary correlates with spelling when given to the right hemisphere but correlates with something else when given to the left. Indeed, many possibilities of this kind exist, and only a multivariate approach can resolve the issue.

\section{Method}

\section{Previous Analyses and Procedure}

Some analyses of the data to be presented here have already been reported by Stankov and Spilsbury (1978). For that reason details regarding procedure will be brief. The aim of the experiment was to replicate Stankov's previous findings about the factorial structure of auditory abilities. The aim was also to find out whether there were any differences between sighted, partially sighted, and blind children (degree of vision - "between" main effect) and right-, left-, and both-ear presentation of stimuli (condition of presentation- "within" main effect). The $F$ tests for both main effects were found to be significant using multivariate analysis of variance, but interaction between them was not significant. For that reason two separate reports have been prepared.

The sample consisted of 30 subjects for each between-groups condition-90 subjects altogether. ${ }^{2}$ Blind and partially sighted children were taken from two specialized schools in the Sydney metropolitan area. On the basis of their medical examination, they were screened for any other physical deficiency, including hearing. (Additional data regarding the sample can be found in Table 1.) Children were tested in groups of 7 to 11 on 2 consecutive days during the normal school hours. The whole battery lasted approximately 4 school periods. The order of the tests was the same for all groups because, under conditions of prolonged testing, it was important to disperse "more interesting" tests among the "boring" ones. This helped to ensure very good rapport.

All tests were recorded on tape and the same tape recorder was used to present them. Of the 26 tests, 19 were presented under the following three conditions. Following the instructions, a

\footnotetext{
${ }^{2}$ Notice that this sample includes practically the entire population of blind children of that age from Sydney (about $3,000,000$ inhabitants altogether).
} 
Table 1
Some Descriptive Statistics for our Sample
$(\mathrm{N}=90)$

\begin{tabular}{lcccc}
\hline & & Sex & \multicolumn{1}{c}{$\begin{array}{c}\text { HQ } \\
\text { Age }\end{array}$} & $\begin{array}{c}\text { Handed- } \\
\text { ness }\end{array}$ \\
\hline Mean & 13.41 & .70 & 103.00 & .11 \\
$\begin{array}{l}\text { Standard Deviation } \\
\text { Intercorrelations : } \\
\quad \text { Age }\end{array}$ & 1.39 & .46 & 17.54 & .31 \\
Sex & 1.0 & -.14 & -.13 & .00 \\
IQ & -.14 & 1.0 & -.04 & -.08 \\
$\quad$ Hand. & -.13 & -.04 & 1.0 & .08 \\
\hline
\end{tabular}

complete set of items (e.g., 21 for a given test) was presented with the first block of 7 going to both channels, the next block of 7 to the left, and the last block to the right. This procedure was not followed in those tests where items were ordered with respect to difficulty (Tests 6 and 13). In these cases the first item was given to both, the second to left, and the third to right ear, and so forth. It is possible that fixed order of presentation might have had some effect, but it is not likely that the effect would be very pronounced. Children were told that the ear of presentation would change but that they should disregard this and take the test in a normal way. Seven tests, which were not included in the main analysis below, were given only under two of the above three conditions. ${ }^{3}$

Blind children recorded their answers using Braille typewriters, whereas the other two groups wrote their answers on separate sheets of paper containing test title and item numbers. $A$ typical item in the battery consisted of a stimulus, and then a short break of about 2

${ }^{3}$ In an effort to replicate the results reported here, some additional data have been collected. This time, special care was taken to change some of the procedural aspects of the present study. In particular, the order of presentation of listening conditions was varied, and the same items were used for all three listening conditions. Unfortunately, the analyses of these new data have not been completed. seconds. This was followed by presentation of alternatives with 1 -second intervals between them, then a 7- to 10-second break for recording answers, and so forth. This kind of presentation puts considerably more demand on immediate memory than was the case in earlier studies, but that did not seem to affect the children's ability to work on the tasks.

\section{Variables and Scoring}

A short description of the tests used is given below. The numbers in parentheses indicate the test's number in Stankov and Spilsbury's (1978) report."

1.2. (4-5)

3. (6) (21 items).

Reordering. Assign numbers to the three notes (letters), and write the order they occur in the second presentation. Response: 312,231 , and so forth

Tonal Figures. Choose the set of notes containing the same four notes as in the first set but played in the reverse order.

${ }^{4}$ Test titles correspond to those used by Stankov and Horn (in press), who provided a more detailed description of the tests. 
Four alternatives from which to choose (21 items).

4-5. (7-8) Series. Which of the three notes (chords) continues the sequence established in first four? Three alternatives from which to choose (21 items).

6. (9) Vocabulary. Subjects choose synonym of a spoken word from among five alternatives. Words range from easy to difficult (24 items).

7. (10) Cloze. Write two words to complete an eight-word sentence. Score: Number of correct words (21 sentences).

8. (11) Rapid Spelling. Words spelled rapidly. Subjects write the word spelled. Blind subjects allowed to use contractions (21 items).

9. (12) Chord Analysis. (Wing, 1961). How many notes played in a chord? (15 items).

10. (13) Pitch Change (Wing, 1961). Two chords played in which one note may differ. State whether chords are the same or if the changed note moved up or down (21 items).

11. (14) Tonal Memory (Wing, 1961). Pairs of tunes up to 10 notes long were presented. Which note has changed in the second playing? (21 items).

12. (15) Chord Decomposition. Choose a set of three individually played notes which comprised a chord (21 items).

13. (16) Tonal Memory (Seashore, Lewis, \& Satveit, 1960) Pairs of sequences are presented. Each sequence consists of three, four, or five tones. Which note differs in the second playing? (21 items).
14. (19)

Chord Parts Decomposition. A chord composed of three notes was presented. After this, three alternatives were given. Each alternative was a two-note interval. One of the alternatives involved two notes from the first chord. Subjects had to indicate this alternative (21 items).

15. (19) Sound Blending. Word sounded part by part. Choose the word from among four phonetically balanced words. Score: Number correct (21 items).

16-17. (20-21) Expanded and Compressed Speech. Word was played at slower (faster) speed. Identify word from among four choices (21 items).

18. (24) Rhythm (Seashore et al., 1960). Are two rhythmic patterns the same or different? (21 items).

19. (26) Tempo "B' (Drake, 1954). Same as (25) below except that the interfering beat was introduced in place of the silent interval.

The following tests were given under two (not all three) conditions of presentation:

(1-2) Memory Span. Tests from French et al. (1963).

(3) Dichotic Listening. Six digits given in pairs, three to the left ear and three to the right ear at two rates of presentation (one pair per second and two pairs per second). Write down digits in order, giving left ear first, then right ear (18 items).

(17) Melodies (Drake, 1954). Match subsequent melodies with the first melody and indicate if they are the same, if they are in a different key, or if a note or time is different (10 items, 40 answers). 
(22-23) White Noise and Talk Masking. Pick out single word from continuous background talking (or noise). Choose one of four alternatives, spoken later, as being the same as masked word (35 items).

(25) Tempo "A" (Drake, 1954). Subjects are to continue to count a beat established by a metronome during silence until told to stop. Score: Number of beats different from norms (10 items).

\section{Analyses}

The analyses to be reported here were carried out using the Little Jiffy, Mark IV (LJIV) program for factor analysis (Kaiser \& Rice, 1974). Little Jiffy is based upon image analysis and orthoblique rotations and employs a root-one criterion for determining the number of factors to be retained. This method was chosen for two reasons. First, almost 20 different empirical correlational matrices (including Stankov, 1978) were analyzed by both LJIV and Jöreskog's (1966) Unrestricted Maximum Likelihood Factor Analysis (UMLFA). These show that for the same number of factors, the Promax rotated factor pattern from UMLFA is very similar to the factor pattern matrix from LJV. Secondly, factor intercorrelations from LJIV are exactly the same as correlations between the factor scores. This second property is crucial for obtaining the result of Table 4 below.

LJIV was used in order to obtain a hierarchical solution along the lines proposed by Schmid and Leiman (1957). ${ }^{5}$ This solution is based upon the "fundamental theorem of factor analysis" and is obtained in two phases. The first (forward) phase involves the analysis of factor intercorrelation matrices at successive orders until either an orthogonal solution or one factor is obtained. The second (backward) phase, starts from the highest order and proceeds downward by first augmenting the factor pattern matrix

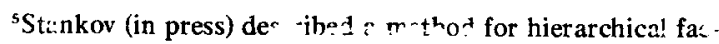
toring with Little Jiffy. with the diagonal matrix of uniquenesses, then premultiplying this new matrix with the factor pattern matrix of the lower order, augmenting this with the uniquenesses matrix of the same order, premultiplying this matrix with the pattern matrix of the next lower order, and so forth.

Since LJIV is based upon image analysis, it can never be used to obtain a proper SchmidLeiman solution. To illustrate this and to point out the modifications employed, consider the "fundamental equation of image analysis" (Mulaik, 1972):

$R=G-Q+s^{2}$

The four $n \times n$ matrices above are correlational matrix (R); image covariance matrix $(\mathbf{G})$; antiimage covariance matrix $(\mathbf{Q})$; and anti-image variance matrix $\left(\mathbf{S}^{2}\right)$, which is diagonal. LJIV's factor pattern matrix $A$ of the order $n \times m$ reproduces $\mathbf{G}$, i.e.,

$\mathrm{G}=\mathrm{AR}_{1} \mathrm{~A}^{\prime}$

where $\mathbf{R}_{1}$ is an $m \times m$ factor intercorrelation matrix. This latter matrix was further analyzed along the lines of the forward phase of the Schmid-Leiman solution. Notice, however, that in doing this, Equation 2 has to change, since the second-order factor pattern matrix $\mathbf{A}_{1}$ also reproduces $G_{1}$, not $R_{1}$, and the same happens at the higher order.

Matrix $\mathbf{S}^{2}$ was treated as analogous to the uniqueness matrix. At every order of factoring, this matrix was obtained by subtracting a diagonal matrix containing squared multiple correlations from an identity matrix. It is obvious that in going from one order to the next the antiimage covariance matrix $\mathbf{Q}$ is disregarded. This can have a serious effect on the final result; but provided that LJIV's measures of sampling adequacy at every order are reasonable, the loss is likely to be negligible. It should be mentioned that there are probably several other possibilities for obtaining hierarchical solutions with LJIV.

The results reported in Tables 2 and 3 were obtained in the manner indicated above. How- 
ever, those of Table 4 were obtained as follows: If $\mathbf{R}$ is considered as a supermatrix of the order $(l+r) \times(l+r)$, where $l$ and $r$ indicate the number of variables belonging to two separate subgroups $(l+r=n)$, then

$$
R=\left[\begin{array}{c|c}
11^{R} & 1 r^{R} \\
\hline r 1^{R} & r r^{R}
\end{array}\right]
$$$$
\mathrm{n} \times \mathrm{n}
$$

Separate analyses of ${ }_{\text {}} \mathbf{R}$ and ${ }_{r r} \mathbf{R}$ can be carried out and $\mathbf{A}$ and $\mathbf{S}^{2}$ matrices of Equations 1 and 2 can be presented in terms of the following supermatrices:

$$
\begin{aligned}
& \mathrm{A}=\left[\begin{array}{l|r}
1^{\mathrm{A}} & 0 \\
\hline 0 & \mathrm{r}^{\mathrm{A}}
\end{array}\right] \\
& (1+r) \quad x\left({ }_{1} m_{1}+{ }^{m} m_{1}\right)
\end{aligned}
$$

$$
\operatorname{dgS}^{2}=\left[\begin{array}{c|c}
1^{2} & 0 \\
\hline 0 & \mathrm{r}^{2}
\end{array}\right]
$$

where ${ }_{l} m_{1}$ and ${ }_{r} m_{1}$ represent the number of factors obtained from $l$ and $r$ sets at the first order. The factor intercorrelation matrix $\mathbf{R}_{1}$ can be obtained as a correlation matrix of factor scores and can be seen as another supermatrix:

$$
R_{1}=\left[\begin{array}{l|l}
I 1^{R_{1}} & 1 r^{R_{1}} \\
\hline r 1_{1}^{R_{1}} & \mathrm{rr}^{R_{1}}
\end{array}\right]
$$

With a proper strategy, factor scores can be used to obtain factor intercorrelations, not only between the first-order factors, but between factors of any order.

Let it be assumed that the analysis of $\mathbf{R}_{1}$ gives either two orthogonal factors or only a single factor and that the second-order factor pattern matrix and corresponding anti-image variance matrix are

$$
A_{1}=\underbrace{\left[\frac{r^{A_{1}}}{r^{A_{1}}}\right]}_{\left({ }_{1}{ }_{1}+m_{1}\right)} \times \mathrm{m}_{2}
$$

and

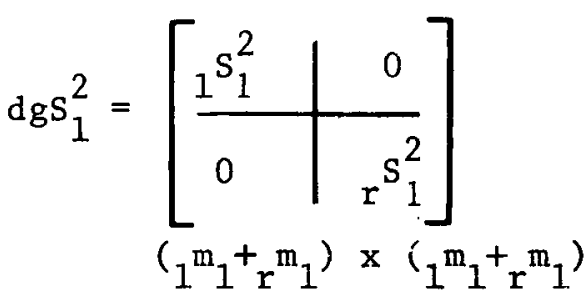

At the "backward" stage $A_{1}$ and $S_{1}$ can be augmented and the resulting matrix premultiplied with $\mathbf{A}$, as required by the Schmid-Leiman procedure.

In this case the last matrix consists of two parts. The first part shows the loadings of all variables on the second-order factor(s) and the second part shows the separate loadings of the $l$ set of variables on the $l$ factors and the $r$ set of variables on the $r$ factors. It is obvious that there can be a different number of variables in the $l$ and $r$ sets and that the variables can be grouped into more than two sets.

\section{Results}

\section{Ear Differences in Terms of Factor Loadings}

It has been found by Stankov and Spilsbury (1978) that factor analysis of the total scores for all 26 tests produced seven factors, six of which were the same as those in Stankov's earlier studies. This result is important in its own right, since it shows that the factor structure based on the free-room testing conditions is virtually identical to the factor structure of the sum of the both-, left-, and right-ear conditions employed here. It is not, as might have been expected, 
identical with the analysis of the scores for the both-ears condition.

The seven factors found in that study were as follows:

1. Auditory Immediate Memory (Msa)

2. Temporal Tracking (Tc)

3. Auditory Cognition of Relationships (ACoR)

4. Tonal Memory (Tm)

5. Speech Perception Under Distraction/Distortion (SPUD)

6. Maintaining and Judging Rhythm (MaJR)

7. Spelling Specific

Since 19 rather than 26 variables were used here, it would be expected that the reduction in the size of test battery would change the factor structure. On the basis of the above findings, it can be claimed that the total-score analysis of the 19 variables represents the "true" factor structure. The aim of this study, then, was to attempt to compare the factors obtained from separate analyses of both-, left-, and right-ear scores with these "true" total-scores factors.

The proposition seems fairly straightforward: The intention was to compare the first-order factors obtained from total scores with those obtained from the separate matrices for both-, left-, and right-ear conditions. However, the first-order solutions from these four matrices differed: The total-scores matrix gave four factors, whereas each of the other matrices yielded six factors. All 22 factors were rather dissimilar. Intuitively, this does not seem likely because the total scores were based on the both-, left-, and right-ear scores, and greater similarity would have been expected from this comparison.

There are several approaches available to further elucidate the relationships among the factors. The theory underlying the following development rests on the assumption that higher order factors, because of their breadth, will show a greater overlap between the conditions of presentation.
On the basis of the Little Jiffy analyses, three hierarchical solutions were obtained. These are presented in the next three sections in Tables 2 to 4.

Factor analysis of total scores- "true" structure. Factor analysis of the correlation matrix obtained from total scores is given in Table 2. Loadings marked with asterisks are first-order salients from Little Jiffy. Three of the four firstorder factors are Tm (Factor II), SPUD (Factor III), and MaJR (Factor IV). These are the primaries which defined $\mathrm{Ga}$ in previous studies.

The first factor is obviously a broad factor defined by markers for Tc and ACoR, although the loadings which Vocabulary, Cloze, and Rapid Spelling have on it also imply Verbal Comprehension. It is apparent that with exclusion of 7 variables from the battery of 26 , the following results were obtained: Three factors remained the same, one factor (Memory Span) disappeared, the Spelling specific shared its variance between SPUD and broad Factor I, and two factors (Tc and ACoR) merged. The broad Factor I represents intellective processes, largely of the Gf variety, but with some evidence of Gc. The second-order factor is obviously broad enough to have loadings from all tests of the battery.

Correlated condition-specific factors. This solution assumed that specific factors for every condition of presentation can be correlated with any other specific factor. It operated on the entire $57 \times 57$ supermatrix ( $3 \times 19$ variables). With this solution, analysis had to be carried to the third order. (The first-order solution involving 17 factors is not reproduced in Table 3). From 17 factors, 4 were extracted at the second order and one at the third. Second- and third-order factors are arranged in this table so that the loading from both-, left-, and right-ear conditions can be seen. To be viewed correctly, they should be seen as $57 \times 1$ vectors (this also holds for the results of Table 4).

It can be seen from Table 3 that the thirdorder factor had loadings from all variables and that, overall, none of the conditions exceeded 
Table 2

'True' Factor Structure

Hierarchical Solution Obtained

with Total Scores

\begin{tabular}{|c|c|c|c|c|c|c|c|}
\hline & \multirow{2}{*}{ Variable } & \multirow{2}{*}{$\begin{array}{c}2 \text { nd } \\
\text { Order }\end{array}$} & \multicolumn{4}{|c|}{ First-Order Factors } & \multirow[b]{2}{*}{$\mathrm{R}^{2}$} \\
\hline & & & $I$ & II & III & IV & \\
\hline 1 & Tonal Reordering & .46 & $.37 *$ & .12 & -.04 & -.01 & .52 \\
\hline 2 & Letter Reordering & .59 & $.48 *$ & -.08 & .06 & -.12 & .71 \\
\hline 3 & Tonal Figures & .35 & .17 & .11 & .16 & .10 & .43 \\
\hline 4 & Tonal Series & .46 & $.45 *$ & .26 & $-.25 *$ & .02 & .66 \\
\hline 5 & Chord Series & .44 & .31 & $.31 *$ & -.15 & .04 & .56 \\
\hline 6 & Vocabulary & .30 & $.57 *$ & -.12 & -.11 & .09 & .48 \\
\hline 7 & Cloze & .62 & $.53 *$ & .07 & .12 & -.02 & .78 \\
\hline 8 & Spelling & .49 & .24 & .02 & $.38 *$ & .16 & .59 \\
\hline 9 & Chord Analysis & .41 & $-\overline{-.22}$ & $.48 *$ &.$\overline{.20}$ & .01 & .59 \\
\hline 10 & Pitch Change & .37 & -.05 & $.60 *$ & -.11 & .05 & .59 \\
\hline 11 & Tonal Memory (wing) & .57 & -.06 & $.49 *$ & .11 & -.08 & .68 \\
\hline 12 & Chord Decomposition & .32 & .01 & $.47 *$ & .04 & $-.22 *$ & .43 \\
\hline \multirow[t]{2}{*}{13} & Tonal Memory & & & & & & \\
\hline & (Seashore) & .58 & .01 & $.44 *$ & .01 & -.17 & .67 \\
\hline \multirow[t]{2}{*}{14} & Chord Parts & & & & & & \\
\hline & Decomposition & .35 & .20 & .33 & -.11 & .08 & .38 \\
\hline 15 & Sound Blending & .54 & .03 & .03 & $.50 *$ & -.01 & .66 \\
\hline 16 & Expanded Speech & .46 & .12 & .04 & $.37 *$ & .06 & .51 \\
\hline 17 & Compressed speech & .44 & -.05 & .01 & $.28 *$ & $.26 *$ & .45 \\
\hline 18 & Seashore's Rhythm & .43 & .06 & .10 & -.01 & $.33^{*}$ & .43 \\
\hline 19 & Drake's Tempo 'B' & .40 & .08 & -.01 & .06 & $.44 *$ & .50 \\
\hline
\end{tabular}

* Salient loadings from the first-order Little Jiffy. All loadings above .20 are underlined.

the others in the size of the third-order loadings. Average loadings for all three conditions were very close.

Much more interesting are the loadings of the second-order factors. They can conveniently be compared with the first-order factors of Table 2 and were measured through all three conditions employed here. The last two second-order factors of Table 3 show some resemblance to SPUD and MaJR, but these (especially the last one) were relatively poorly defined.

In order to conserve space, the first-order solution has not been reproduced here. The 17 factors that were found can be roughly divided into two groups: (1) those which had loadings on the same test across all conditions and (2) mixed cases.

Correlated first-order common factors. This solution was similar to the previous one in that it required computation of the third-order factor, but it did not allow for correlations among the condition-specific factors; only common-factor correlations were permitted to affect the secondand third-order structure. It has already been mentioned that every matrix at the first order produced six condition-specific factors-18 factors altogether. Factor scores were then intercorrelated. This yielded four second-order factors 


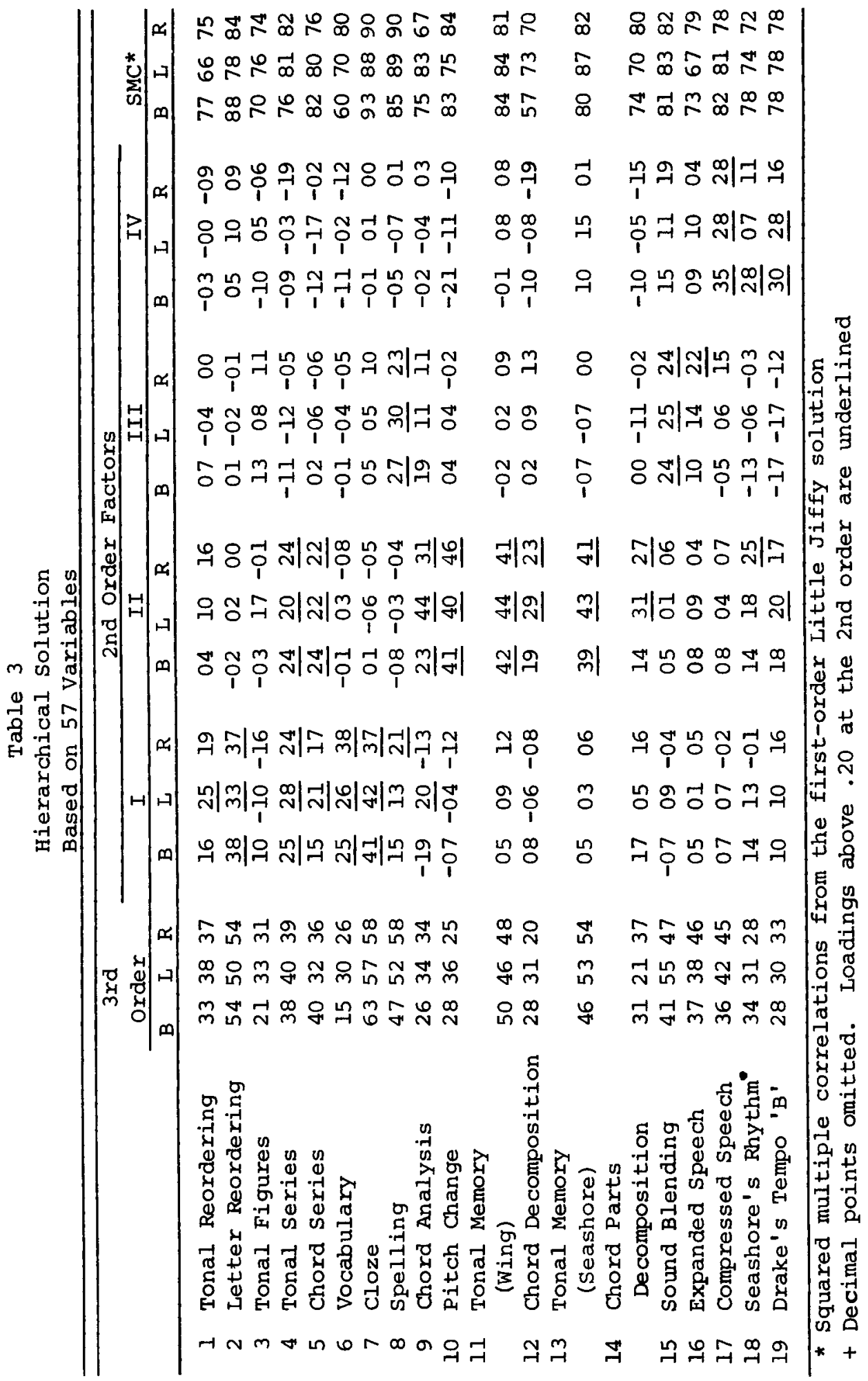




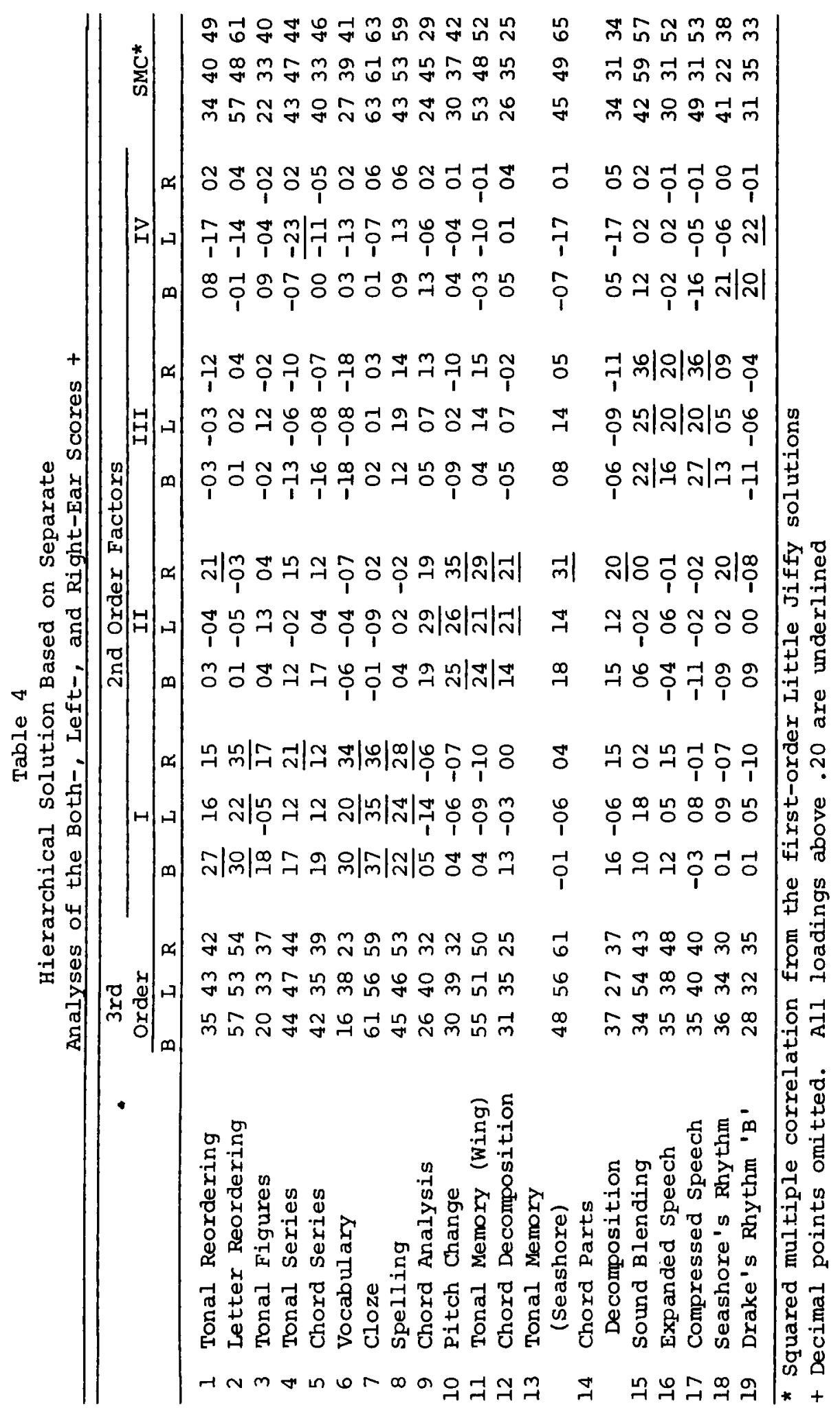


from which one third-order factor was extracted. Table 4 presents the hierarchical solution which is comparable to that of Table 3.

The third-order factor of Table 4 is very similar to the corresponding factor from Table 3. Two second-order factors of Table 4 can be clearly seen as Tm (Factor II) and SPUD (Factor III). Factor IV is a MaJR (but with loadings from right-ear scores absent). Of all the factors obtained with the present data, Factor I had the closest resemblance to Verbal Comprehension. It is interesting that when auditory tests were presented to the right ear, no MaJR secondorder factor occurred with this solution. This may be of some importance, but an attempt at explanation should probably await further evidence.

The 18 first-order factors are not presented here. They are either condition specific or similar to some of the second-order factors.

Conclusions regarding factorial structure. In summary, the attempt to express a "true" matrix in terms of the three conditions of presentation showed that it cannot be expected that similar first-order factors would be found across the conditions but that hierarchical factoring could be used to achieve this. Furthermore, the agreement with previous findings is considerable. Present results, of course, should be interpreted cautiously and in accordance with other limiting features of the experiment. For example, a $57 \times$ 57 matrix based on 90 subjects can be viewed with some suspicion. It is rather comforting, therefore, that the solution of Table 4 produced similar results to the earlier study.

Although it is true that factors of the second order corresponded closely to the total scores primary factors, it was possible to compare the results of Tables 3 and 4. This comparison showed that Table 4 provided a somewhat better approximation of the "true" matrix.

\section{Ear Differences in Terms of Differences Between the Means}

In this section the problems that concern experimental psychologists interested in hemi- spheric lateralization will be considered. The question is whether or not there will be significant differences between the mean vectors for scores on those parts of the tests presented to both, left, and right ears. The hypothesis was that there would be no differences, since functional decussation was not allowed to operate to its maximum; there was no dichotic listening employed. Yet, some of the suggestions mentioned before indicated that it would be important to check on that hypothesis.

Table 5 presents means, standard deviations, and $F$ tests for the difference between both-, left-, and right-ear (B-, L-, and R) scores for each of the 19 tests. From this table it can be seen that $12 F$ tests were significant at the .05 level. It should be kept in mind that a significant $F$ test in the present situation may have arisen not only because of the left- versus right-ear differences but also because of the both-ears condition. In order to clarify this, Table 6 was prepared.

In Table 6 only the 12 variables with significant $F$ tests are included. For these variables a protected $t$ test was used to compare every possible pair of conditions. The first column presents the ordering of the means. The next three columns utilize information from the first column and indicate the direction of the differences from pairwise comparisons. This breakdown is provided because hemispheric differences are typically derived from the left- versus right-ear differences (given in the last column of Table 6).

It can be seen that the right ear was superior to left ear on Vocabulary, Letter Reordering, and Rapid Spelling. All these tests used speech material and showed left hemispheric superiority, as expected. Both Vocabulary and Rapid Spelling are measures of Verbal Comprehension (V), which, at the higher order, measure Gc.

Letter Reordering, on the other hand, represents a measure of $\mathrm{Tc}$ and Gf. Another test which had a loading on Tc in Stankov and Spilsbury's (1978) study was Drake's Melodies test. This test was given to the left and right ear only 
Table 5

Means, Standard Deviations and F-Tests

of the Differences between the

conditions of Presentation

\begin{tabular}{|c|c|c|c|c|c|c|c|c|}
\hline \multirow{3}{*}{\multicolumn{2}{|c|}{ Variable }} & \multirow[b]{2}{*}{ Both } & \multirow[b]{2}{*}{ Ears } & \multirow[b]{2}{*}{ Left } & \multirow[b]{2}{*}{ Ear } & \multirow{2}{*}{\multicolumn{2}{|c|}{ Right Ear }} & $F$ \\
\hline & & & & & & & & $\mathrm{df}_{1}=2,174$ \\
\hline & & $\bar{x}$ & SD & $\overline{\mathrm{X}}$ & SD & $\bar{x}$ & SD & Fcrit $=3.9$ \\
\hline 1 & Tonal Reordering & 2.10 & 1.47 & 1.83 & 1.34 & 2.02 & 1.41 & 1.50 \\
\hline 2 & Letter Reordering & 3.69 & 1.92 & 2.84 & 1.65 & 3.34 & 2.34 & $11.48 *$ \\
\hline 3 & Tonal Figures & 2.10 & 1.54 & 2.14 & 1.36 & 1.92 & 1.25 & .94 \\
\hline 4 & Tonal Series & 3.57 & 1.68 & 3.56 & 1.54 & 3.31 & 1.70 & 1.31 \\
\hline 5 & Chord Series & 2.84 & 1.69 & 3.38 & 1.52 & 3.21 & 1.63 & $4.43 *$ \\
\hline 6 & Vocabulary & 1.97 & 1.43 & 1.78 & 1.33 & 2.56 & 1.45 & $10.81 *$ \\
\hline 7 & Cloze & 6.96 & 3.44 & 7.57 & 3.31 & 7.50 & 3.47 & 3.84 \\
\hline 8 & Spelling & 3.53 & 1.96 & 3.40 & 2.25 & 4.37 & 2.04 & $25.80 *$ \\
\hline 9 & Chord Analysis & 2.04 & 1.26 & 2.94 & 1.36 & 2.44 & 1.32 & $19.41 *$ \\
\hline 10 & Pitch Change & 2.53 & 1.39 & 2.86 & 1.42 & 3.01 & 1.38 & $5.12 \star$ \\
\hline 11 & Tonal Memory (Wing) & 2.79 & 1.58 & 2.89 & 1.35 & 2.52 & 1.29 & $3.94 *$ \\
\hline 12 & Chord Decomposition & 2.48 & 1.45 & 2.94 & 1.49 & 2.87 & 1.40 & $3.93 *$ \\
\hline 13 & $\begin{array}{l}\text { Tonal Memory } \\
\text { (Seashore) }\end{array}$ & 5.96 & 1.44 & 4.73 & 1.81 & 3.81 & 2.19 & $77.27 *$ \\
\hline 14 & Chord Parts & & & & & & & \\
\hline & Decomposition & 3.67 & 1.57 & 3.80 & 1.49 & 3.93 & 1.61 & 1.20 \\
\hline 15 & Sound Blending & 6.17 & 1.19 & 5.47 & 1.51 & 5.61 & 1.60 & $12.79 *$ \\
\hline 16 & Expanded Speech & 6.38 & .99 & 5.74 & 1.30 & 5.93 & 1.38 & $9.44^{*}$ \\
\hline 17 & Compressed Speech & 5.52 & 1.34 & 5.76 & 1.40 & 5.78 & 1.41 & 1.84 \\
\hline 18 & Seashore's Rhythm & 6.39 & .90 & 6.02 & 1.18 & 5.83 & 1.13 & $9.46 *$ \\
\hline 19 & Drake's Tempo 'B' & 5.04 & 3.48 & 4.82 & 3.46 & 5.56 & 3.73 & 2.19 \\
\hline
\end{tabular}

* Significant at .05 level.

and showed right-ear superiority, although it does not use speech but musical material. Tonal Reordering also showed a similar trend, although the difference was not significant. It can be said, therefore, that $V$ and $T c$ were performed better by the left hemisphere.

Turning to the tests where a left-ear superiority was evident, it can be seen that these were Seashore's Tonal Memory test and Wing's tests of Chord Analysis and Tonal Memory-all of which measure the primary factor $\mathrm{Tm}$ and the second-order factor Ga. All these showed the same trend in the studies of Milner (1962), Kimura (1967), and Gordon (1974).

It can be concluded that the primary factors showing ear differences are V, Tc, and Tm. No ear differences exist for ACoR, SPUD, and MaJR. Memory Span tests were given through left and right ear but not through both ears, and they also showed no differences. (The Dichotic Listening test, which loads on the same factor, showed differences, as expected.)

Since it is claimed here that left- versus rightear differences are not the only differences of 
Table 6

Ordering of the Means for those

Variables of Table 5 which showed

Significant Differences

\begin{tabular}{|c|c|c|c|c|c|}
\hline \multirow{2}{*}{\multicolumn{2}{|c|}{ Variable }} & \multirow{3}{*}{$\frac{\text { Order* }}{\mathrm{B}>\mathrm{R}>\mathrm{L}}$} & \multicolumn{3}{|c|}{ Pairwise Comparisons } \\
\hline & & & \multirow{2}{*}{$\frac{\mathrm{B}-\mathrm{L}}{>}$} & \multirow{2}{*}{$\frac{\mathrm{B}-\mathrm{R}}{>}$} & \multirow{2}{*}{$\frac{L-R}{<}$} \\
\hline 2 & Letter Reordering & & & & \\
\hline 5 & Chord Series & $\mathrm{L}=\mathrm{R}>\mathrm{B}$ & $<$ & $<$ & $=$ \\
\hline 6 & Vocabuiary & $\mathrm{R}>\mathrm{B}=\mathrm{L}$ & $=$ & $<$ & $<$ \\
\hline 8 & Spelling & $\mathrm{R}>\mathrm{B}=\mathrm{L}$ & $=$ & $<$ & $<$ \\
\hline 9 & Chord Analysis & $\mathrm{L}>\mathrm{R}>\mathrm{B}$ & $<$ & $<$ & $>$ \\
\hline 10 & Pitch Change & $\mathrm{R}=\mathrm{L}>\mathrm{B}$ & $<$ & $<$ & $=$ \\
\hline 11 & Tonal Memory (Wing) & $\mathrm{I}=\mathrm{B}>\mathrm{R}$ & $=$ & $>$ & $>$ \\
\hline 12 & Chord Decomposition & $\mathrm{L}=\mathrm{R}>\mathrm{B}$ & $<$ & $<$ & $=$ \\
\hline \multirow[t]{2}{*}{13} & Tonal Memory & & & & \\
\hline & (Seashore) & $B>L>R$ & $>$ & $>$ & $>$ \\
\hline 15 & Sound Blending & $B>I=R$ & $>$ & $>$ & $=$ \\
\hline 16 & Expanded Speech & $B>R=L$ & $>$ & $>$ & $=$ \\
\hline 18 & Seashore's Rhythm & $B>L=R$ & $>$ & $>$ & $=$ \\
\hline
\end{tabular}

significance in our understanding of human abilities, the results from the both-ears condition will be discussed in the next section.

\section{Discussion}

\section{Possible Nature of General Auditory Function}

It has already been mentioned that $\mathrm{Gv}$ and $\mathrm{Ga}$ are assumed to represent broad perceptual factors and that one of the important features of $\mathrm{Gv}$ is its localization in the right hemisphere. $\mathrm{Ga}$, as has been seen, does not admit to such a localization.

One hypothesis, which will be examined here, states that competition between auditory messages is basic to the understanding of $\mathrm{Ga}$ and that such competition usually results in an im:pairment of normal cognitive functioning. Competition can occur at the level of sensory organs, along the afferent pathways and within or between the hemispheres. It can be caused either by simultaneous or successive arrival of the messages.

In order to illustrate this, attention here will be focused on primary factors, not the variables which defined them. With SPUD the task is relatively simple, since this factor is measured by tests involving mutilated words. Of course, the kind of mutilation differs from one particular test to another, but in all cases speech is made to sound more like nonspeech, and competition occurs with regard to the locus of processing between the hemispheres. With MaJR, it can be assumed that competition occurs between the internally regulated rhythmic processes and externally introduced tempo or rhythmic patterns.

The third Ga factor, Tm, cannot be seen as involving competition without further complicating the whole picture. First, the role of binaural stimulation will be examined. In general, pos- 
sibly because of an increased amount of information-reaching centers, binaural stimulation shows superiority over monaural. The first column of Table 6 shows that for 5 out of 12 variables, binaural presentation was superior. In three cases the binaural condition was intermediate between the left- and right-ear conditions, and in four cases the binaural presentation was the poorest. Until further replications of these results are obtained, it is probably advisable to postpone serious consideration of the binaural condition being placed in the middle. However, it is useful to have a closer look at the three $\mathrm{Tm}$ tests for which the binaural condition showed the lowest mean.

Chord Analysis, Chord Series, and Pitch Change were not the only tests for which the binaural mean was the lowest. Two tests not among the 19 showed a similar trend: White Noise and Talk Masking. In both of these, the task was to choose from among four phonetically balanced words-the one previously spoken against the masking background. These two tests consistently measured the SPUD primary factor and shared some variance with $G f$ and $\mathrm{Gc}$ factors. These tests were given under several conditions, by varying ear of presentation of signal and mask. The finding of some significance for the argument is that the worst performance occurred when masking material was given binaurally.

Turning now to the three Tm tests, it can be seen that all of them involved chords. The other tests of the battery also involving chords (Tonal Decomposition, Chord Parts Decomposition) showed exactly the same trend; the binaural condition showed the worst result, but the difference was not significant. It can be claimed that the phenomenon known within the area of musical perception as binaural diplacusis (musical paracusis) was responsible for the inferior binaural performance. In binaural diplacusis the same tones, especially those at higher frequencies, given to two ears give rise to perception of different pitches. This may result not only when one ear is not "normal" but also when they both have normal sensitivity (see Ward, 1970). It appears that this effect is more pronounced when chords are given instead of single tones, and that the results of this study can be attributed to it. What is important here is that the binaural result tells about competition between the auditory messages coming from different ears. It is this competition that was hypothesized as being responsible for the emergence of Ga.

\section{Conclusions Regarding Lateralization}

The results showed that primary factors of $\mathrm{V}$ and Tc demonstrated right-ear and left-hemisphere lateralization and that $\mathrm{Tm}$ was better performed by the left ear and right hemisphere. All other primary auditory factors did not show ear differences. A hypothesis is put forward that lateralization is not typical of $\mathrm{Ga}$ but that competition between auditory messages on their way to the cortical centers is typical. Competition can be due to the mutilated nature of auditory input, differences between internal and external rhythms, or a binaural listening peculiarity called binaural diplacusis.

\section{Summary and Conclusions}

The aim of research reported here was to explore the nature of several primary auditory factors defining broader higher order factors in the Gf and Gc theory. Of particular interest was the difference in terms of both factor structure and mean vectors between the tests presented to both, left, and right ears and, by implication, between the two hemispheres.

Before looking at ear differences, it was necessary to establish if the present battery measured the same factors as it did in previous studies. Despite changes in subjects (the present sample differed from previous ones with respect to age, culture, language, and degree of visual impairment) and procedure (previous studies employed free-room condition and imposed less demand on memory), the agreement is remarkable. Total 
scores on 26 tests produced six out of seven factors obtained earlier with the same battery. These were Msa, Tc, ACoR, Tm, SPUD, and MaJR. A reduced battery of 19 tests produced a Gf factor (composed of markers for ACoR and Tc) plus the last three factors from the above list. These three represent a measure of $\mathrm{Ga}$.

The evidence, therefore, shows that factors obtained in a free-room situation are the same as factors obtained from scores representing the sum of left-, right-, and both-ear conditions. This should be the case, since in a free-room situation listening occurs with both ears but (because of phase and intensity differences) there are also different signals in each ear. This situation appears to be very simple indeed, and yet it is not discernable from consideration of factors obtained from the analysis of the left-, right-, and both-ears scores separately. The reason is that the analysis of the total scores approximates only higher order factors, and this is true irrespective of whether there is allowance for correlations between the condition-specific factors or only common factors. With this in mind, it can be said that the same abilities are measured by either left, or right, or both ears and that differences occur only at the lower order of factoring.

Provided that it can be demonstrated in the future that the present results did not follow from some idiosyncracies of the correlational matrix, hierarchical factoring appears to be a very useful method for studying lateralization; and it is quite possible that its potential application is much wider than is typically recognized.

At the planning stage of this research, it was thought that the study of hemispheric differences would help substantiate the claim that $\mathrm{Ga}$ is a broad perceptual factor like $\mathrm{Gv}$. It is believed by the author that this has been achieved, but in a different way from what was originally anticipated. It should be understood that $\mathrm{Ga}$ is different from $\mathrm{Gv}$ because only a part of $\mathrm{Ga}$ (Tm) is localized at the right hemisphere; and if some suggestions are taken seriously, even that part can be shifted to the other side (see Bever \&
Chiarello, 1974; Locke \& Kellar, 1973). The results show that some findings (Robinson \& Solomon, 1973) regarding rhythm's left-hemisphere localization may be lacking in support, since no differences were found. This applies to SPUD variables as well. It is hypothesized that localization is not typical of $\mathrm{Ga}$ but that competition between the auditory messages is. This competition is assumed to take place before "higher mental processes" are actualized, and in this sense it has a perceptual quality.

There were no surprises regarding the localization of Gf and Gc. One Gc primary factor (V), and probably others as well, is located to the left. There is no reason to think that differences should be found between the hemispheres regarding Gf primaries unless the verbal (e.g., Letter Reordering) or visualization (see Zaidel \& Sperry, 1973) component is overwhelming.

It is obvious that not all abilities show lateralization. 'This is true of abilities being measured by the present battery, and it will probably be the case with many other abilities reported elsewhere. Of course, lateralization should always be checked, since in this way it can be found whether a primary ability is likely to represent $\mathrm{Gc}$ or Gv. With the information on the both-ears condition, it is possible to distinguish in some cases between $\mathrm{Ga}$ and the other three broad factors. This means that lateralization may be seen as an additional source of information, which can be helpful in the understanding of ability structure.

\section{References}

Arnold, E. M. Temporal integration as a dimension of intellect. Unpublished doctoral dissertation, University of Sydney, Australia, 1964.

Bever, T. G., \& Chiarello, R. Cerebral dominance in musicians and nonmusicians. Science, 1974, 185 , 537.

Blumstein, S., \& Cooper, W. E. Hemispheric processing of intonation contours. Cortex, 1974, 10, 146-159.

Bock, D. R. Word and image: Source of the verbal and spatial factors in mental test scores. Psychometrika, 1973, 38, 437-458. 
Cattell, R. B. Abilities: Their structure, growth, and action. Boston: Houghton Mifflin, 1971.

Darwin, G. J. Ear differences in the recall of fricatives and vowels. Quarterly Journal of Experimental Psychology, 1971, 23, 46-62.

Darwin, G. J. Ear differences and hemispheric specialization. In F, O. Schmitt \& F. G. Worden (Eds.), The neurosciences. Third study program. Cambridge, MA: M.I.T. Press, 1974.

Drake, R. M. Manual for Drake Musical Aptitude Tests. Chicago: University of Chicago Press, 1954.

French, J. W., Ekstrom, R. B., \& Price, L. A. Manual for kit of reference tests for cognitive factors, Princeton, NJ: Educational Testing Service, 1963.

Gordon, H. W. Hemispheric asymmetries in the perception of musical chords. Cortex, 1970, 6, 387.

Gordon, H. W. Hemispheric asymmetry and musical performance. Science, 1974, 189, 68-69.

Haydon, S. H., \& Spellacy, J. F. Monaural reaction time asymmetries for speech and non-speech sounds. Cortex, 1973, 9, 288-295.

Hearnshaw, L. S. Temporal integration and behaviour. Bulletin of the British Psychological Society, $1956,30,1-20$.

Horn, J. L. Organization of abilities and the development of intelligence. Psychological Review, 1968, 75, 242-259.

Horn, J. L. Organization of data on life-span development of human abilities. In R. Goulet \& P. B. Baltes (Eds.), Life-span developmental psychology. New York: Academic Press, 1972.

Hunt, E., Lunneborg, C., \& Lewis, J. What does it mean to be high verbal? Cognitive Psychology, 1975, 7, 194-227.

Jöreskog, K. G. Some contributions to maximum likelihood factor analysis (RB-66-41). Princeton, NJ: Educational Testing Service, 1966.

Kaiser, H., \& Rice, P. Little Jiffy, Mark IV. Educational and Psychological Measurement, 1974, 37, 111-117.

Karlin, J. E. Music ability. Psychometrika, 1941, 6, 61-65.

Kimura, D. Functional asymmetry of the brain in dichotic listening. Cortex, 1967, 3, 163-178.

Locke, S., \& Kellar, L. Categorical perception in a nonlinguistic mode. Cortex, 1973, 9, 353-367.

Milner, B. Laterality effects in audition. In V. B. Mountcastle (Ed.,), Interhemispheric relations and cerebral dominance. Baltimore, MD: John Hopkins Press, 1962.

Milner, B. Hemispheric specialization: Scope and limits. In F. O. Schmitt \& F. G. Worden (Eds.), The neurosciences. Third study program. Cambridge, MA: M.I.T. Press, 1974.

Mulaik, S. A. The foundation of factor analysis. New York: McGraw-Hill, 1972.
Roberts, L. D., \& Gregory, A. H. Ear differences and delayed auditory feedback. Journal of Experimental Psychology, 1973, 101, 269-272.

Robinson, G. M., \& Solomon, D. J. Rhythm is processed by the speech hemisphere. Journal of Experimental Psychology, 1973, 102, 508-511.

Schmid, T., \& Leiman, T. M. The development of hierarchical factor solutions. Psychometrika, 1957, 22, 53-63.

Seashore, C.E., Lewis, D., \& Saetveit, J. O. Manual of instructions and interpretation for the Seashore Measures of Musical Talents (2nd ed.). New York: Psychological Association, 1960.

Shankweiler, D., \& Studdert-Kennedy, M. Identification of consonants and vowels presented to left and right ears. Quarterly Journal of Experimental Psychology, 1967, 19, 59-68.

Stankov, L. The hierarchical structure of auditory abilities and the relationship between auditory and visual modalities. Unpublished doctoral dissertation, University of Denver, 1971.

Stankov, L. Fluid and crystallized intelligence and broad perceptual factors among the 11-12 year olds. Journal of Educational Psychology, 1978, 70, 324-334.

Stankov, L. Hierarchical factoring based on image analysis and orthoblique rotations. Multivariate Behavioral Research, in press.

Stankov, L., \& Horn, J. L. Human abilities revealed through auditory tests. Journal of Educational Psychology, in press.

Stankov, L., \& Spilsbury, G. Auditory abilities of blind, partially sighted, and sighted children. $A p$. plied Psychological Measurement, 1978, 2, 491-503.

Ward, M. Musical perception. In J. V. Tobias (Ed.), Foundations of modern auditory theory (Vol. 1). New York: Academic Press, 1970.

Wing, H. Manual for Standardized Tests of Musical Intelligence. Sheffield, England: City of Sheffield Training College, 1961.

Zaidel, D., \& Sperry, R. W. Performance on the Raven's Coloured Progressive Matrices Test by subjects with cerebral commissurotomy. Cortex, $1973,9,134-40$.

\section{Acknowledgments}

I am grateful to Georgina Spilsbury for collecting the dat a for the present study.

\section{Author's Address}

Send requests for reprints or further information to Lazar Stankov, Department of Psychology, University of Sydney, Sydney, N.S.W. 2006, Australia. 